\title{
Influência de um Ordenhador Aversivo sobre a Produção Leiteira de Vacas da Raça Holandesa
}

\author{
Maria José Hötzel ${ }^{1}$, Luiz Carlos Pinheiro Machado Filho ${ }^{2}$, Maria Cristina Yunes ${ }^{3}$, Marcela \\ Cristina A. C. da Silveira ${ }^{4}$
}

\begin{abstract}
RESUMO - Neste estudo, foi testada a hipótese de que o medo de seres humanos é duradouro e pode prejudicar a produção leiteira de vacas da raça Holandesa. Um tratador aversivo realizou duas sessões diárias de um tratamento hostil (duas palmadas na região posterior e um grito forte), durante 21 dias, e um tratador neutro ordenhou as vacas, durante o mesmo período, não oferecendo nenhuma ameaça. A distância de fuga na presença do tratador aversivo e do neutro foi medida antes e 14 e 180 dias após o início do tratamento. As produções total e residual de leite (após aplicação i.v. de ocitocina na veia caudal) foram medidas a partir da ordenha realizada pelo tratador aversivo ou ou pelo neutro, entre os dias 14 e 24 do experimento. Todas as vacas $(n=13)$ participaram dos dois tratamentos, constituindo um delineamento experimental do tipo “cross-over". Com exceção do período anterior ao experimento, as vacas mantiveram maior distância de fuga do tratador aversivo que do neutro, mesmo 180 dias após o último contato com os tratadores. Entretanto, não houve efeito do tratamento sobre a produção total ou residual de leite. Os resultados corroboram outros estudos que comprovaram que vacas discriminam tratadores aversivos de neutros. Entretanto, o tratamento aqui aplicado não afetou as produções total ou residual de leite.
\end{abstract}

Palavras-chave: bem-estar animal, bovino, etologia, medo, relação humano-animal

\section{An Aversive Milker Causes Fear, But Does Not Influence Milk Yield of Holstein Cows}

\begin{abstract}
In the present study the hypothesis that fear of humans is long-lasting and may influence milk production in Holstein cows was tested. One person (the aversive handler) carried out two daily sessions of an aversive treatment during 21 days, where each cow received two slaps on the rump and a strong yell. The control handler walked around the cows not menacing the cows. Flight distance from both handlers was measured before the treatments were imposed and 14 and 180 days later. Total and residual milk (measured after an i.v. oxytocin injection in the caudal vein) were measured when either the aversive or the neutral handler carried out the milking, between days 14 and 24 of the experiment. All the cows were used in both treatments in a cross-over design. Except for the day before treatments started, the cows kept a larger distance from the aversive than from the neutral handler, even after 180 days without contact with these handlers. Nevertheless, there was no effect of treatment on total milk yield or residual milk. The results corroborate others that show that cows can discriminate between aversive and neutral individuals. Under the conditions of the present study, the fact that milking was done by the aversive individual did not influence total milk yield and residual milk.
\end{abstract}

Key Words: bovine, ethology, animal welfare, human-animal interactions, fear

\section{Introdução}

As relações entre o homem e os animais podem ter grande impacto sobre o bem-estar de animais de fazenda, constituindo-se um aspecto da criação animal em que, com educação, é possível alcançar boas mudanças sem grandes custos, melhorando os níveis do bem-estar animal (Hemsworth et al., 2003).

Originariamente presas, os ruminantes ainda mantêm suas características comportamentais primitivas que os levam à detecção e fuga de predadores. $\mathrm{O}$ medo tem importante papel neste processo, ao moti- var os animais a evitarem situações potencialmente perigosas (Boissy, 1995). O ser humano pode causar medo aos animais em virtude de seu tamanho e sua propensão a desenvolver movimentos rápidos e imprevisíveis. Os efeitos do medo na fisiologia se refletem, por sua vez, em resultados negativos na produção animal, especialmente por meio da intensificação da atividade do eixo hipotálamo-hipófiseadrenal, expressos em aumentos no cortisol, e do sistema nervoso simpático, pelo aumento nas concentrações de adrenalina e dos batimentos cardíacos (Hemsworth, 2003).

\footnotetext{
1 Professor Visitante, Laboratório de Etologia Aplicada, Departamento de Zootecnia e Desenvolvimento Rural; Universidade Federal de Santa Catarina; Rodovia Admar Gonzaga, 1346, Itacorubi; Florianópolis, SC, Brazil. CEP: 88.034-001 (mjhotzel@cca.ufsc.br).

2 Professor Adjunto, Laboratório de Etologia Aplicada, Departamento de Zootecnia e Desenvolvimento Rural; Universidade Federal de Santa Catarina; Rodovia Admar Gonzaga, 1346, Itacorubi; Florianópolis, SC, Brazil. CEP: 88.034-001 (pinheiro@cca.ufsc.br).

3 Bióloga, Mestranda em Agroecossistemas, DZ/UFSC.

4 Médica Veterinária, Mestranda em Agroecossistemas, DZ/UFSC.
} 
Segundo Seabrook (1994), vacas sob tratamento aversivo na sala de ordenha defecaram seis vezes mais que aquelas tratadas gentilmente, sugerindo ativação do sistema nervoso simpático. Em outro experimento, vacas que receberam maus tratos ao entrarem na sala de ordenha, algo que freqüentemente ocorre na prática, apresentaram redução na produção de leite em relação àquelas tratadas gentilmente (Breuer et al., 2000). Hemsworth et al. (1995) compararam a produção leiteira de 14 propriedades leiteiras na Austrália, e sujeitaram as vacas a um simples teste de medo, medido com base na distância que as vacas mantinham do experimentador durante o teste. Além da forte correlação negativa entre a distância mantida pela vaca e a produção leiteira da propriedade, os autores concluíram que de 30 a 50\% da variação na produção leiteira entre propriedades foi explicada pelo nível de medo das vacas por seres humanos. Além de uma correlação negativa entre a freqüência de maus tratos na sala de ordenha e a produção leiteira, Hemsworth et al. (2000) encontraram forte correlação positiva com a concentração de cortisol no leite.

Rushen et al. (1999) expuseram vacas sistematicamente a um tratador aversivo e a um tratador gentil. Após uma semana, realizaram a ordenha com a presença do tratador aversivo ou do tratador gentil, ou com a ausência de ambos. A presença do tratador aversivo durante a ordenha aumentou em $70 \%$ o leite residual, medido depois da ordenha após injeção intravenosa de ocitocina, que libera o leite retido nos alvéolos da glândula mamária. O leite residual foi medido porque aumenta em situações de estresse, possivelmente em decorrência da liberação de oxitocina endógena (Rushen et al., 2001). Trabalhos posteriores também indicaram que vacas discriminam um tratador gentil de um aversivo (Munksgaard et al., 2001; Machado Filho et al., 2001). Entretanto, segundo Machado Filho et al. (2001), a presença do tratador aversivo durante a ordenha per se não influenciou o leite residual das vacas em relação à do tratador gentil, sugerindo que, nas condições desse estudo, talvez fosse necessária a manipulação durante a ordenha para causar medo às vacas.

Este experimento foi desenvolvido dentro das mesmas condições experimentais do trabalho realizado por Machado Filho et al. (2001), para testar a hipótese de que quando a ordenha é realizada por um tratador considerado aversivo, a quantidade de leite retido no úbere após a ordenha é maior que quando a ordenha é feita por um tratador neutro. Adicionalmente, investigou-se a capacidade de vacas leiteiras em diferenciar o tratador aversivo de um tratador neutro seis meses após o tratamento.

\section{Material e Métodos}

O trabalho foi realizado na Unidade de Gado Leiteiro do Centro de Treinamento da EPAGRI (Empresa de Pesquisa Agropecuária e Extensão Rural de Santa Catarina), em Florianópolis, SC.

Treze vacas leiteiras da raça Holandesa, em diferentes estádios de lactação, foram utilizadas no experimento. As vacas apresentavam produção leiteira média de aproximadamente 30 litros/dia e, em sua rotina, tinham acesso a pasto pela manhã e à noite $\mathrm{e}$ recebiam silagem e ração concentrada diariamente. Todo o manejo era feito por três funcionários que trabalham no CETRE há alguns anos. A ordenha era feita diariamente com ordenhadeira mecânica por um dos três tratadores, às $5 \mathrm{~h} 30 \mathrm{e} 17 \mathrm{~h}$. A rotina foi mantida durante o experimento.

Antes da ordenha da manhã do primeiro dia, as vacas foram pintadas com tinta atóxica, para identificação. Houve, então, 21 dias de manejo "aversivo", com duas sessões por dia, que consistiram de dois tapas de cada lado da garupa e um grito a cada tapa. Vacas apresentam mais medo de gritos que de choques elétricos (Pajor et al., 2003), razão pela qual este tratamento foi considerado aversivo. Este tratador vestia camiseta vermelha e usava chapéu, uma vez que vacas tendem a associar cores (não necessariamente a cor vermelha) e vestimentas ao tipo de tratamento (Rybarczyk et al., 2003). As sessões foram impostas em horários variados, não coincidentes com a ordenha, no curral de espera, onde as vacas permaneciam oito horas por dia comendo silagem e ração. O controle consistiu da presença de um tratador neutro, ou seja, que não promovia estimulação ativa positiva ou negativa - nos animais, na mesma freqüência, mas em horários distintos do tratamento aversivo. Este tratador vestia sempre camiseta branca e não usava chapéu.

Entre os dias 21 e 180 do experimento, as vacas foram submetidas à sua rotina normal e não tiveram contato algum com ambos os tratadores.

Testes de distância de fuga foram realizados, para se avaliar a capacidade de cada vaca em reconhecer 
os tratadores e evitar o tratador aversivo. A distância de fuga foi definida como a distância mínima de aproximação permitida pela vaca, antes de iniciar um deslocamento (Hurnik et al., 1995).

Os testes foram realizados no pasto, em três ocasiões: na véspera do início do tratamento, quando ambos os tratadores eram desconhecidos das vacas; aos 14 e aos 180 dias após o início do tratamento. $\mathrm{O}$ tratador entrava no piquete e se aproximava de cada vaca lentamente, sem emitir som ou gesticular e anotava a distância de fuga. Como a distância de fuga nunca foi maior que $5 \mathrm{~m}$, foram consideradas as seguintes possibilidades: distância $0=$ quando a vaca deixava ser tocada, distância $1=$ aproximação de 0 $1 \mathrm{~m}$, distância 2 = aproximação de $1 \mathrm{a} 2 \mathrm{~m}$, distância 3 = aproximação de 2 a $3 \mathrm{~m}$, distância 4 = aproximação de 3 a $4 \mathrm{~m}$, distância $5=$ aproximação de mais de $4 \mathrm{~m}$.

Cada teste de distância de fuga foi realizado duas vezes por dia. Em cada teste, um tratador por vez entrava no piquete, em ordem sorteada e alternada. Entre a saída de um tratador e a entrada do outro, aguardava-se 5 minutos e entre um teste e outro, 10 minutos. Na análise foi utilizada a média por vaca por tratamento por dia dos dois testes.

As vacas foram agrupadas por paridade, estágio de lactação e produtividade e divididas aleatoriamente em dois grupos, um de seis e outro de sete vacas. Cada grupo foi exposto a um tratamento por vez. Aos 14 dias do experimento, as vacas do grupo 1 foram ordenhadas pelo indivíduo aversivo e as do grupo 2 , pelo indivíduo neutro. Aos 17 dias, o mesmo procedimento foi repetido. Aos 21 e aos 24 dias, o segundo teste foi realizado em igual procedimento, apenas invertendo-se o tratamento que cada grupo recebeu. Os tratadores estavam presentes na sala de ordenha somente quando estavam ordenhando as vacas a eles alocadas.

Nos dias de coleta, as vacas eram manejadas na ordenha pelos funcionários de rotina. $\mathrm{O}$ tratador, vestindo as mesmas roupas utilizadas durante o tratamento, permanecia parado, encostado na parede em frente ao cubículo da respectiva vaca ordenhada, em silêncio, imóvel, com as mãos no bolso e sem fitar a vaca, até que esta fosse presa pelo tratador de rotina. A partir deste momento, todo o procedimento de ordenha era realizado pelo tratador. As vacas entravam na sala de ordenha, onde eram isoladas visualmente do restante do grupo, aos pares, sempre na mesma ordem. Toda a ordenha (contenção, lavagem do úbere, aplicação das teteiras e desinfeção das tetas) era realizada individualmente.

Foram registradas a produção de leite e a quantidade de leite residual de cada vaca, no dia das coletas. Dois minutos após a retirada das teteiras, era feita uma única aplicação de 20 UI de ocitocina na veia mediana caudal. O leite residual era extraído por ordenha mecânica um minuto após a injeção de ocitocina.

O protocolo experimental foi aprovado pelo Comitê de Experimentação Animal da Universidade Federal de Santa Catarina (CEUA), que atende as recomendações do Colégio Brasileiro em Experimentação Animal.

O desenho experimental utilizado foi um crossover, onde todas as vacas participaram de ambos os tratamentos. A vaca foi considerada a unidade experimental, totalizando, assim, 13 repetições. Os efeitos do tratamento na produção leiteira e no leite residual foram submetidos à análise de variância. Os efeitos de tratamento sobre a distância de fuga foram analisados por meio de análise de variância com medidas repetidas no tempo (Snedecor \& Cochran, 1989). No modelo, foram incluídos os fatores fixos tratamento e dia. Este último não afetou significativamente $(\mathrm{P}<0,05)$ nenhuma variável e, portanto, foi removido do modelo. As análises foram realizadas com o auxílio do programa SuperAnova (BrainPower Inc., Berkeley) e os resultados são apresentados como média \pm desvio-padrão.

\section{Resultados e Discussão}

Houve interação significativa entre período e tratamento para a distância de fuga mantida pelas vacas em relação aos tratadores $(\mathrm{P}<0,001)$. Antes do início do experimento, as vacas dos dois tratamentos mantiveram a mesma distância de fuga dos dois tratadores. Entretanto, nos dias 14 e 180 do experimento, a distância de fuga foi maior em relação ao tratador aversivo que ao tratador neutro $(\mathrm{p}<0,01$; Figura 1$)$. Ou seja, após a imposição do tratamento, as vacas evitaram mais o indivíduo aversivo que o neutro. O teste de distância de fuga é considerado confiável para avaliar o medo que bovinos sentem de seres humanos e tem sido aplicado por diversos pesquisadores com essa finalidade (Seabrook \& Bartle, 1992; Hemsworth et al., 1995; Rushen et al., 1999; Breuer et al., 2003; Waiblinger et al., 2003). No caso de animais presos, 
como os bovinos, o teste representa muito bem uma situação natural, em que, em vista da aproximação de um predador, o animal se desloca para uma distância segura.

Estes resultados estão de acordo com os de outros estudos que comprovam que as vacas possuem capacidade de distinguir as pessoas pelas quais foram tratadas, discriminando tratadores aversivos de neutros (Rushen et al., 1999; Munksgaard et al., 2001; Machado Filho et al., 2001). Neste trabalho, após 14 dias de tratamento, enquanto a distância de fuga aumentou em relação ao tratador aversivo, diminuiu em relação ao tratador neutro. O comportamento das vacas em relação ao tratador neutro sugere um processo de habituação, em que as vacas "aprenderam" que este indivíduo nunca as ameaçava ou thes oferecia perigo.

Ressalta-se que, após 180 dias, mesmo sem ter mantido contato com nenhum dos dois tratadores, as vacas ainda mantiveram maior distância do tratador aversivo que do neutro. Se, por um lado, este achado não é surpreendente, considerando, por exemplo, que ovelhas reconhecem as faces de um grande número de outras ovelhas por um período semelhante (Kendrick et al., 2001), tem relevância do ponto de vista prático. Alguns procedimentos de rotina que são percebidos como aversivos pelos animais como atendimentos veterinários e vacinações, podem ser associados à pessoa que os aplica e ser reconhecidos pelos animais por longos períodos. Se a mesma pessoa também estiver envolvida em outras atividades com

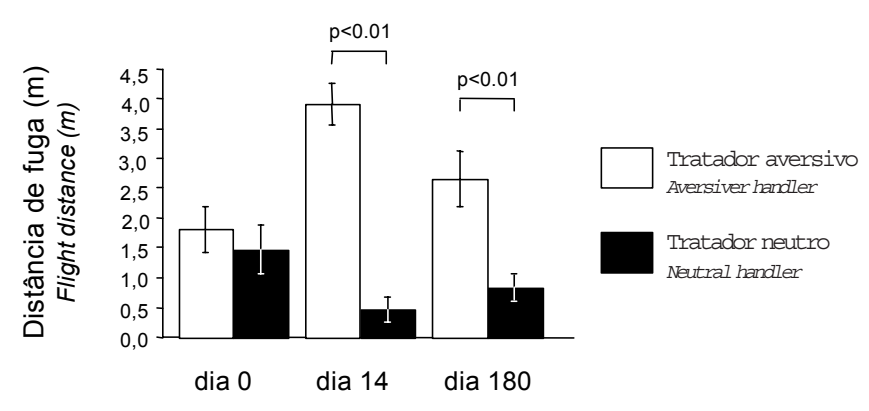

Figura 1 - Distância de fuga das vacas em relação ao tratador neutro ou aversivo, nos dias $0,14 \mathrm{e}$ 180 do experimento.

Figure 1 - Flight distance of the cows kept from the aversive or from the neutral handler, on days 0, 14 and 180 of the experiment. os mesmos animais, o que não é incomum, isto pode dificultar manejos não-aversivo ou resultar em inquietação por sua presença. Há evidências de que o medo que vacas sentem durante procedimentos veterinários pode ser reduzido por um tratamento adequado e pela presença de um tratador gentil (Waiblinger et al., 2004). Maiores conhecimentos sobre aspectos que levam os animais a interpretarem tratamentos ou tratadores como aversivos e sobre a generalização das experiências negativas ocorridas com pessoas e em locais específicos são importantes para indicar melhorias nas relações entre tratadores e animais durante o manejo.

Vacas parecem utilizar principalmente informações visuais, como a face e altura ou porte do indivíduo, no reconhecimento de seres humanos, sendo que o odor parece não contribuir (Rybarczyk et al., 2001). Suínos também parecem utilizar informações visuais no reconhecimento de seres humanos em conjunto com informações auditivas, enquanto o odor contribui em conjunto com outras, mas tem pouca importância isoladamente (Tanida \& Nagano, 1998). Também tem sido sugerido que vacas utilizam-se da cor das roupas para discriminarem diferentes tratadores (Munksgaard et al., 1999), além de, em algumas situações, identificarem dois tratadores vestindo a mesma cor (Rybarczyk et al., 2001). Entretanto, em situações em que o tratador impõe medo, é possível que o animal não consiga concentrar suas atenções em detalhes, reconhecendo somente características mais gerais, como a cor da roupa (Munksgaard et al., 1999; Mendl, 1999). Neste experimento, os tratadores utilizaram vestimentas de cores diferentes para se reforçar a capacidade de discriminação das vacas.

A hipótese de que a ordenha realizada pelo tratador aversivo resultaria em maior produção de leite residual não foi confirmada neste experimento. Não houve efeito de tratamento sobre a produção de leite $(\mathrm{P}=$ 0,9 ; Figura 2) ou sobre a quantidade de leite residual coletado das vacas $(\mathrm{P}=0,7$; Figura 3$)$. Os resultados para o leite residual contrariam aqueles reportados por Rushen et al. (1999) e Breuer et al. (2000), que registraram aumento de até $70 \%$ no leite residual das vacas submetidas à presença de um tratador aversivo. Nenhuma tendência foi observada neste experimento para estas duas variáveis, embora, ao contrário de Rushen et al. (1999), tenha-se evidenciado que todas as vacas diferenciaram os indivíduos. Por outro lado, em outros estudos, também não houve diferenças no 
leite residual quando um tratador aversivo esteve presente durante a ordenha, embora as vacas tenham reconhecido o tratador (Munksgaard et al. 2001; Machado Filho et al., 2001).

Alguns fatores, como o fato de o local onde foi imposto o tratamento não ter sido o mesmo onde foi feita a medição do leite residual, podem explicar estas discrepâncias. Rushen et al. (1998) demonstraram que vacas reconhecem mais claramente tratadores neutros ou aversivos no mesmo local onde recebem o tratamento. Neste experimento, como no de Machado Filho et al. (2001), o tratamento foi aplicado na sala de espera onde as vacas passavam parte do dia, enquanto a medição do estresse pela quantidade de leite residual ocorria na sala de ordenha. Embora a distância de fuga tenha sido medida no pasto, também um local diferente daquele onde o tratamento foi imposto, as vacas reconheceram claramente os diferentes tipos de tratadores, o que enfraquece esta explicação.

A experiência anterior dos animais com seres humanos, possivelmente relacionada a diferenças nos sistemas criatórios, pode ser a explicação mais plausível para justificar as discrepâncias entre os estudos, encontradas nas respostas do leite residual. Por exemplo, o sistema de criação e a experiência prévia com seres humanos influenciam a resposta ao manejo em bezerros (Lensink et al., 2001) e vacas (Raussi, 2003).

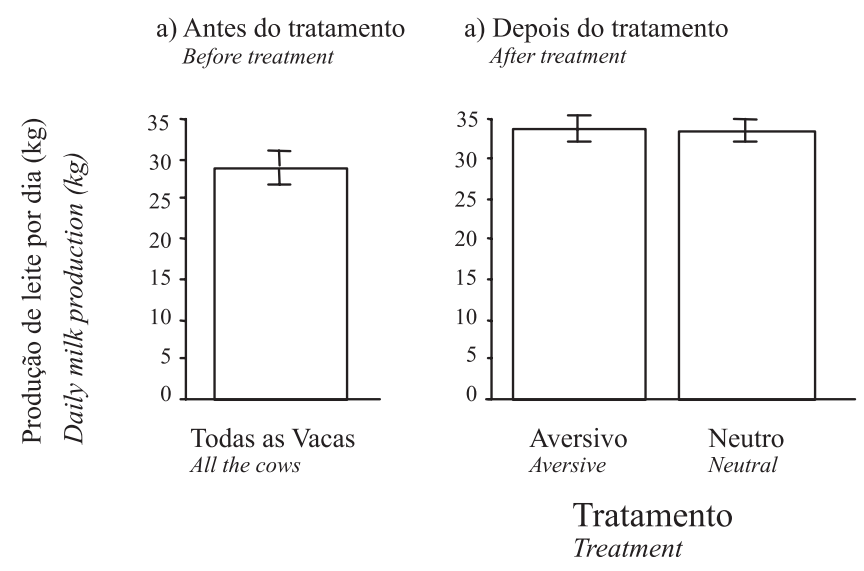

Figura 2 - Produção total diária de leite a) antes e b) após o tratamento.

Figue 2 - Total daily milk production a) before and b) after aversive treatment.
O tamanho do rebanho, que foi maior nos estudos citados que neste trabalho, também tem influência na qualidade das relações humano-animais (Waiblinger \& Menke, 1999). Por último, Breuer et al. (2003) mostraram que novilhas generalizam os maus tratos recebidos por um tratador, refletindo-se em reações de medo e estresse fisiológico em resposta à presença de outras pessoas desconhecidas. O contrário também pode ser verdadeiro. Estudos já demonstraram que interações positivas entre seres humanos e vacas podem diminuir o estresse causado por procedimentos veterinários de rotina, como palpação retal e inseminação artificial (Waiblinger et al., 2004). O manejo e a criação das vacas utilizadas nos experimentos realizados no mesmo laboratório diferem muito daqueles de produção intensiva, onde existe grande mecanização e há poucas oportunidades de interações humanas positivas. As vacas utilizadas neste experimento e no de Machado Filho et al. (2001) estavam acostumadas a interações positivas com humanos, possivelmente diminuindo o medo e reagindo melhor a situações estressantes (Mendl, 1999). No trabalho de Rushen et al. (1999), as vacas foram contidas na ordenha e no estábulo, o que pode ter feito com que generalizassem a experiência do estábulo para a sala de ordenha, pois, em ambas as situações, não podiam fugir. Neste trabalho, entretanto, em todas as vezes que a vaca foi ameaçada,

\section{Leite Residual (ml)} Residual Milk (ml)

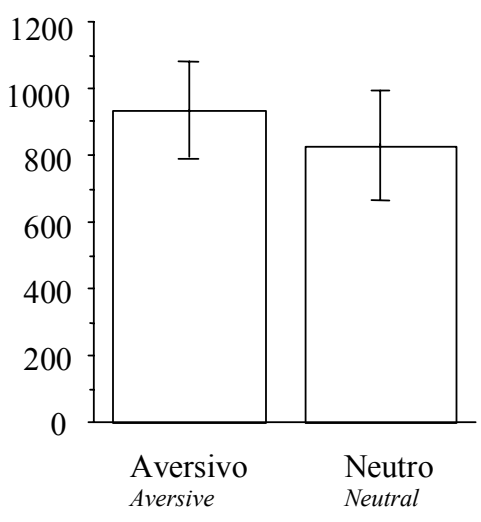

Tratamento Treatmento

Figura 3 - Leite residual $(\mathrm{mL})$ após a ordenha realizada com o tratador aversivo e neutro.

Figure 3 - Residual milk $(m L)$ after the milking done by the aversive or the neutral handler. 
houve possibilidade para fuga, podendo esta superar o estresse, em uma "resposta de neutralização" ao estímulo aversivo. Isto talvez tenha condicionado a presença do tratador aversivo a uma situação de impotência diante do fator estressor, o que, por sua vez, resulta no fenômeno da desistência aprendida (learned helplessness), condição extremamente estressante, em que há uma "resposta de resignação", caracterizada por cessar a resistência ao estímulo aversivo (Hurnik, 1992).

\section{Conclusões}

Vacas discriminam tratadores aversivos de neutros, condição que permanece por até 180 dias, mesmo quando não há reforço durante mais de cinco meses. Nas condições deste experimento, a ordenha realizada pelo tratador aversivo não afetou a produção de leite total nem a quantidade de leite residual no úbere, indicando que não houve estresse durante a ordenha. As diferenças entre este estudo, realizado em condições de fazenda em sistema de semiestabulação, e outros, desenvolvidas em sistemas de confinamento intensivo, ressaltam a importância de se investigar a influência do sistema de manejo nas interações humano-animais.

\section{Agradecimento}

À Epagri, por permitir a realização do estudo em suas instalações, e aos seus funcionários, pela colaboração nas atividades de rotina. Ao $\mathrm{CNPq}$, pela bolsa $\mathrm{PV}$ concedida à MJH. Aos alunos do curso de Agronomia da UFSC Alexandre Lenzi, Maykol Ouriques e Marcos Alberto Lana, que participaram de algumas atividades.

\section{Literatura Citada}

BOISSY, A. Fear and fearfullness in animals. The Quaterly Review of Biology, v.70, p.165-191, 1995.

BREUER, K.; HEMSWORTH, P.H.; COLEMAN, G.J. The effect of positive or negative handling on the behavioural and physiological responses of nonlactating heifers. Applied Animal Behaviour Science, v.84, p.3-22, 2003.

BREUER, K.; HEMSWORTH, P.; BARNETT, J. et al. Behavioural response to humans and the productivity of commercial dairy cows. Applied Animal Behaviour Science, v.66, n.4, p.273-288, 2000.

HEMSWORTH, P.H. Human-animal interactions in livestock production. Applied Animal Behaviour Science, v.81, p.185-198, 2003.
HEMSWORTH, P.H.; COLEMAN, G.J.; BARNETT, J.L. et al. Relationships between human-animal interactions and productivity of commercial dairy cows. Journal of Animal Science, v.78, p.2821-2831, 2000.

HEMSWORTH, P.H.; BREUER, K.; BARNETT, J.L. et al. Behavioural response to humans and the productivity of commercial dairy cows. In: INTERNATIONAL CONGRESS OF THE INTERNATIONAL SOCIETY FOR APPLIED ETHOLOGY, 29., 1995, Guelph. Proceedings... Guelph: 1995. p.175-176.

HURNIK, J. Behaviour, farm animal and the environment. Cambridge: CAB International, 1992. 430p.

HURNIK, J.F.; WEBSTER, A.B.; SIEGEL, P.B. Dictionary of farm animal behavior. 2.ed. Iowa State University Press: Ames, 1995. 200p.

KENDRICK, K.M.; DA COSTA, A.; LEIGH, A. et al. Sheep don't forget a face. Nature, v.414, p.165-166, 2001.

LENSINK, B.J.; RAUSSI, S.; BOIVIN, X. et al. Reactions of calves to handling depend on housing condition and previous experience with humans. Applied Animal Behaviour Science, v.70, p.187-199, 2001.

MACHADO FILHO, L.C.P.; YUNES, M.C.; HÖTZEL, M.J et al. Is there a relationship between fear of humans and social rank in Holstein cows? In: INTERNATIONAL CONGRESS OF THE ISAE, 35., 2001, Davis. Proceedings... Davis: International Society for Applied Ethology, 2001. p.65.

MENDL, M. Performing under pressure: stress and cognitive function. Applied Animal Behaviour Science, v.65, p. 221 244, 1999.

MUNKSGAARD, L.; DEPASSILLE, A.M.; RUSHEN, J. et al. Dairy cows' fear of people: social learning, milk yield and behaviour at milking. Applied Animal Behaviour Science, v.73, p.15-26, 2001.

MUNKSGAARD, L.; DE PASSILLÉ, A.M.; RUSHEN, J. et al. Dairy cows' use of colour cues to discriminate between people. Applied Animal Behaviour Science, v.65, p.1-11, 1999.

PAJOR, E.A.; RUSHEN, J.; DE PASSILLE, A. M. Dairy cattle's choice of handling treatments in a Y-maze. Applied Animal Behaviour Science, v.80, p.93-107, 2003.

RAUSSI, S. Human-cattle interactions in group housing Applied Animal Behaviour Science, v.80, n.3, p.245-262, 2003.

RUSHEN, J.; MUNKSGAARD, L.; MARNET, P.G. et al. Human contact and the effects of acute stress on cows at milking. Applied Animal Behaviour Science, v.73, p.1-14, 2001 .

RUSHEN, J.; DE PASSILlÉ, A.M.B.; MUNKSGAARD, L. Fear of people by cows and effects on milk yield, behavior, and heart rate at milking. Journal of Dairy Science, v.82, n.4, p.720-727, 1999.

RUSHEN, J.; MUNKSGAARD, L.; DE PASSILLE, A.M. et al. Location of handling and dairy cows' responses to people. Applied Animal Behaviour Science, v.55, p.259-267, 1998.

RYBARCZYK, P.; RUSHEN, J.; DE PASSILlÉ, A.M. Recognition of people by dairy calves using colour of clothing. Applied Animal Behaviour Science, v.81, p.307-319, 2003.

RYBARCZYK, P.; KOBA, Y.; RUSHEN, J. et al. Can cows discriminate people by their faces? Applied Animal Behaviour Science, v.74, p.175-189, 2001.

SEABROOK, M.F. Psychological interaction between the milker and the dairy cow. In: INTERNATIONAL DAIRY HOUSING CONFERENCE ON DAIRY SYSTEMS FOR 
THE $21^{\text {ST }}$ CENTURY, 3., 1994, Orlando. Proceedings... Orlando: ASAE. 1994. p.49-58.

SEABROOK, M.F.; BARTLE, N.C. Human Factors. In: PHILLIPS, C.; PIGGINS, D. (Eds.) Farm animals and the environment. Cab International: Cambridge, 1992. p.111125.

SNEDECOR, G.W.; COCHRAN, W.G. Statistical methods. 8.ed. Iowa State University Press: Ames, 1989. 503p.

TANIDA, H. ; NAGANO, Y. The ability of miniature pigs to discriminate between a stranger and their familiar handler. Applied Animal Behaviour Science, v.56, p.149-159, 1998.

WAIBLINGER, S.; MENKE, C.; KORFF, J. et al. Previous handling and gentle interactions affect behaviour and heart rate of dairy cows during a veterinary procedure. Applied Animal Behaviour Science, v.85, p.31-42, 2004.
WAIBLINGER, S.; MENKE, C. ; FOLSCH, D.W. Influences on the avoidance and approach behaviour of dairy cows towards humans on 35 farms. Applied Animal Behaviour Science, v.84, p.23-39, 2003.

WAIBLINGER, S. ; MENKE, C. Influence of herd size on humancow relationships. Anthrozoos, v.12, p.240-247, 1999.

Recebido em: 16/02/04

Aceito em: 16/04/05 